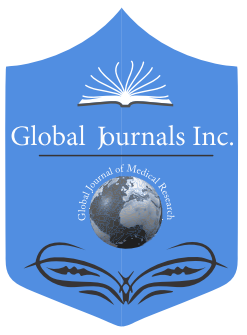

\title{
Effect of RF Fields During Pulse on Rotational Diffusion: Influence on Spectral Density
}

By Dennis J Sorce

Abstract- The effect of the applied RF field in an NMR experiment on the magnitude of the Spectral Density for a Dipolar Relaxation Mechanism is demonstrated theoretically. The effect was shown with $\operatorname{Sin}$ Cos Pulse as a concrete example. The order of magnitude of the magnetic moment where these effects will be significant for typical Rf amplitude values was derived. The effect may be of utility in providing an alternate method of control for MRI Tissue Contrast applications with further development.

GJMR-D Classification: NLMC Code: WN 180

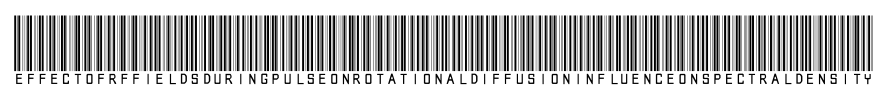

Strictly as per the compliance and regulations of:

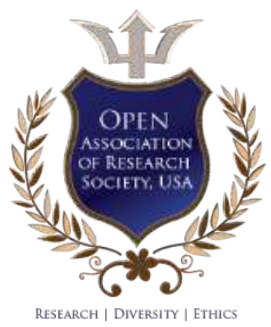

(C) 2020. Dennis J Sorce. This is a research/review paper, distributed under the terms of the Creative Commons AttributionNoncommercial 3.0 Unported License http://creativecommons.org/licenses/by-nc/3.0/), permitting all non-commercial use, distribution, and reproduction in any medium, provided the original work is properly cited. 


\title{
Effect of RF Fields During Pulse on Rotational Diffusion: Influence on Spectral Density
}

\author{
Dennis J Sorce
}

\begin{abstract}
The effect of the applied RF field in an NMR experiment on the magnitude of the Spectral Density for a Dipolar Relaxation Mechanism is demonstrated theoretically. The effect was shown with Sin Cos Pulse as a concrete example. The order of magnitude of the magnetic moment where these effects will be significant for typical Rf amplitude values was derived. The effect may be of utility in providing an alternate method of control for MRI Tissue Contrast applications with further development.
\end{abstract}

\section{INTRODUCTION}

n contemporary NMR methodologies, it is common to find experimental scenarios where the relaxation of the magnetization during a pulse train is important to be able to model and quantify. $(1,2,3)$ In this note, we suggest that for some molecular species the Rotational Diffusion can be affected and modified by the Magnetic Field Torque of the applied radio- frequency pulse. During the course of working on this concept, it has come to our attention that the Russian investigator Sitnitsky (4) has investigated this phenomenon.

This proposed influence may be used in some models for explaining experimental data, such as for Liquid Crystals (14). We demonstrate the derivation of this effect on the spectral densities following the classic treatment of Abragam (5) and gives some ranges of where this effect may be of importance.

We note that the proposed effects may be useful as another avenue to control the spin- dynamics of an experimental system while the pulse is on. Also, the proposed effects have been dealt with rigorously in the Physics Literature (15).

\section{TheOry}

The "Toy Model" we propose to explicate this effect is the following.

We envision a spin system, transformed to the Tilted Doubly Rotating Frame (TDRF,6). In this frame there will be defined a so-called "effective field." We can write down an effective Hamiltonian for the applied RF of the following form:

$$
H^{R F}[t]=I_{x} \omega_{1}[t]+I_{z} \Delta \omega[t]
$$

For the exposition here we consider the Sin/Cos pulse, defined as:

Author: Retired: CMRR, Mn USA 6 Stonegate CtCockeysville. e-mail: dennissorce1@comcast.net

$$
\begin{aligned}
& \omega_{1}[t]=\omega_{1}^{M} \operatorname{Sin}\left[\omega_{1}^{M} t\right] \\
& \Delta \omega[t]=\omega_{1}^{M} \operatorname{Cos}\left[\omega_{1}^{M} t\right]
\end{aligned}
$$

Where $\omega_{1}^{M}$ is a constant (See for example the relevant papers of the Garwood Group $(7,8,9)$.

In the TDRF the effective field can be seen from geometric arguments to be defined as:

$$
\omega_{\text {eff }}[t]=\sqrt{\omega_{1}[t]^{2}+\Delta \omega[t]^{2}}
$$

Substituting Eq [2 a, b] into Eq [3], one easily appreciates that $\omega_{\text {eff }}[t]=\omega_{1}^{M}$. So that as required for our treatment the effective field defined as:

$$
B_{\text {eff }}[t]=\frac{\omega_{1}^{M}}{\gamma_{\text {particle }}}
$$

Where $\gamma_{\text {particle }}$ is the particle gyromagnetic-ratio.

Now we consider a Molecular Species in solution with a defined dipole moment $\mu^{\text {particle }}$.

In a constant field $B_{\text {eff }}$, there is a potential energy of interaction (10) between the moment and the field defined as:

$$
U[\theta]=-\mu^{\text {particle }} B_{e f f} \operatorname{Cos}[\theta]
$$

Here the angle $\theta$ is defined as the angle between the vectorial directions of the dipole moment and the constant field.

We change to the convenient notation:

$$
K_{0}=\mu^{\text {particle }} B_{\text {eff }}
$$

So that:

$$
U[\vartheta]=-K_{0} \operatorname{Cos}[\vartheta]
$$

Knowing the geometry between the effective field and the magnetic moment in the TDRF, it is Seen that the angle $\vartheta$ is defined as:

$$
\vartheta[t]=\operatorname{ArcTan}\left[\frac{\omega_{1}[t]}{\Delta \omega[t]}\right]
$$


Using Eq[2a,b] in Eq[7]we see that:

$$
\vartheta[t]=\omega_{1}^{M} t
$$

Suppose we take the Nuclear Species of Interest to be in a molecule that we model and approximate as a sphere. We assume that the Rotational Brownian Motion can be represented as a series of small incremental rotations. We seek to find the
Correlation Function, which characterizes the rotational diffusion. As treated, in for example Abragam Chapter VIII or other places in the literature $(11,12)$ we can define the Correlation Function in terms of the spatial part of the Dipolar Interaction Hamiltonian. If we adopt the notation of Abragam, we can define the Correlation Function as:

$$
G\left[t, K_{u}, m_{p}\right]=\iint_{\Gamma_{\vartheta} \Gamma_{\vartheta_{p}}} F^{*}\left[\theta, 0, m_{p}\right] F\left[\theta_{p}, 0, m_{p}\right] W\left[\theta, \theta_{p}, K u, t\right] \operatorname{Sin}[\theta] d \theta \operatorname{Sin}\left[\theta_{p}\right] d \theta_{p}
$$

Where we set and consider the case where $m_{p}$ is zero.

So, to carry out this program we need to an expression for the Probability Density Function.

This PDF will be a solution of the so-called Smoluchowski Equation (SE), where the effects of the applied RF Torque will be included. As one can infer there are numerous assumptions one can apply to the formulation of the SE. The solution in general, (see for example, the classic papers of Coffey's group (10) are not trivial, usually the derivation of series solutions which involve the solution of iterative expressions for the expansion coefficients, or continued fraction solutions.

We have chosen to present and use the solution of Sitnitsky (4) which is the most easily implemented solution we have found to program for demonstration of our methods.

Please see Appendix I for a detailed definition of the terms in the series expression for the PDF.

The PDF can be taken to be the approximate solution of the following partial differential Equation using our expression for the Potential Energy Term. (4)

$$
\begin{gathered}
\tau_{R} \frac{\partial w\left[x, x_{i}, K_{u}, t\right]}{\partial t}=\frac{\partial^{2} w\left[x, x_{i}, K_{u}, t\right]}{\partial^{2} x}-\left(2 K_{u}+\tau_{R} C[x]\right) \frac{\partial w\left[x, x_{i}, K_{u}, t\right]}{\partial x}+w\left[x, x_{i}, K_{u}, t\right]\left(K_{u}{ }^{2}+\tau_{R} C[x] K_{u}\right) \\
C[x]=\frac{b}{\operatorname{Cos}\left[k_{1} x\right]} \int_{-1}^{1} d^{\prime} \operatorname{Qos}\left[k_{1} x^{\prime}\right]\left(x-x^{\prime}\right) \operatorname{Exp}\left[-\lambda\left|x-x^{\prime}\right|\right]
\end{gathered}
$$

Here $b$ is a constant defined in (4).

In Eq [10] $\mathrm{x}$ is defined to be as $\operatorname{Cos}[\theta]$ where $K_{u}$ measure as defined previously the interaction between the Moment and the RF field with the definition:

$$
K_{u}=\frac{K_{0}}{k_{B} T}
$$

We can use the definition of the PDF to calculate the Correlation Function as In Eq [9] and then compute the corresponding Spectral Density as:

$$
J\left[\omega, K_{u}, t, m_{p}\right]=\int_{0}^{t} G\left[t_{p}, K_{u}, m_{p}\right] \operatorname{Exp}\left[i \omega t_{p}\right] d t_{p}
$$

In Figure 1 we show the dependence of the Spectral Density as given in Eq [12], for the case $m p=0$.

As can be seen, there is found to be an appreciable dependence of the Spectral Density on the parameter $K_{u}$ this is taken to indicate that the RF Field, with a range of values which will be discussed below, can affect the Spectral Density which is used to compute relaxation functions. $(5,11,12)$ So that the RF field, through interaction on the Rotational Brownian Motion, can influence the values of the calculated relaxation functions during a pulse sequence.
To the knowledge of the author, this possibility has not been fully appreciated in the NMR literature.

\section{ili. Discussion}

The reader may wonder what is a lower bound on the magnetic moment of the particle of Interest for a typical value of the pulse amplitude.

In the Garwood papers $(7,8,9)$, the pulse amplitude is typically on the order of $3.610^{3} \mathrm{~Hz}$. Then we reason that the interaction energy of the magnetic moment with the field in the TDRF should be greater than the thermal energy of the surrounding liquid medium.

So, we propose:

$$
\begin{gathered}
K_{u} \gg 1 \\
u^{\text {particle }} B_{e f f} \gg k_{B} T \\
\text { Or } \\
u^{\text {particle }}>>\frac{k_{B} T}{B_{\text {eff }}}
\end{gathered}
$$

We note that the units of a magnetic moment can be seen in CGS units to be $\frac{\text { ergs }}{\text { Gauss }}$. 
So, at room temperature in CGS units, $k_{B} T$ is on the order of $10^{-14}$ ergs $B_{\text {eff }}$, is typically on the order of $10^{-4}$ Gauss.

$$
u^{\text {particle }}>10^{-10} \frac{\text { ergs }}{\text { Gauss }}
$$

Practical lower bound on the magnetic moment of the particle for an effect of the RF field on the
Rotational Brownian Motion of the particle and consequently on the Spectral Density for a dipolar relaxation mechanism.

\section{ApPENDiX I}

The following is the series definition of the Probability Density Function used in the text. $($ see, 4 )

With

$$
\begin{gathered}
A_{n}=\frac{2 \operatorname{Cos}\left[\frac{\theta_{i}}{2}\right] \operatorname{Sin}\left[q_{n} \operatorname{Cos}\left[\theta_{i}\right]\right]}{\left[1-\frac{1}{2 q_{n}} \operatorname{Sin}\left[2 q_{n}\right]\right]} \\
B_{n}=\frac{2 \operatorname{Cos}\left[\frac{\theta_{i}}{2}\right] \operatorname{Cos}\left[k_{n} \operatorname{Cos}\left[\theta_{i}\right]\right]}{\left[1+\frac{1}{2 k_{n}} \operatorname{Sin}\left[2 k_{n}\right]\right]} \\
\lambda>1
\end{gathered}
$$

$$
\begin{aligned}
& \operatorname{Tan}\left[q_{n}\right]=-\frac{q_{n}}{\lambda} \\
& \operatorname{Cot}\left[k_{n}\right]=\frac{k_{n}}{\lambda} \\
& n=1,2,3, \ldots \ldots .
\end{aligned}
$$

$$
\begin{aligned}
& v_{n}=2 \lambda b \frac{\left[k_{n}^{2}-k_{1}^{2}\right]}{\left[k_{1}^{2}+\lambda^{2}\right]\left[k_{n}^{2}+\lambda^{2}\right]} \\
& \sigma_{n}=2 \lambda b \frac{\left[q_{n}^{2}-k_{1}^{2}\right]}{\left[k_{1}^{2}+\lambda^{2}\right]\left[k_{n}^{2}+\lambda^{2}\right]}
\end{aligned}
$$

$$
w\left[\theta, \theta_{i}, K_{u}, t\right]=\frac{w_{e q}\left[\theta, K_{u}\right]}{\operatorname{Cos}\left[k_{1} \operatorname{Cos}[\theta]\right.}\left[\frac{1}{B_{1}} \sum_{n=1}^{\infty} B_{n} \operatorname{Exp}\left[-v_{n} t\right] \operatorname{Cos}\left[k_{n} \operatorname{Cos}[\theta]\right]+\right.
$$$$
\left.\left(\frac{\operatorname{Cos}\left[k_{1} \operatorname{Cos}\left[\theta_{i}\right]\right]}{\operatorname{Cos}\left[\frac{\theta_{i}}{2}\right] w_{e q}\left[\theta, K_{u}\right]}-\frac{1}{B_{1}}\right) \sum_{n=1}^{\infty} A_{n} \operatorname{Exp}\left[-\sigma_{n} t\right] \operatorname{Sin}\left[q_{n} \operatorname{Cos}[\theta]\right]\right]
$$

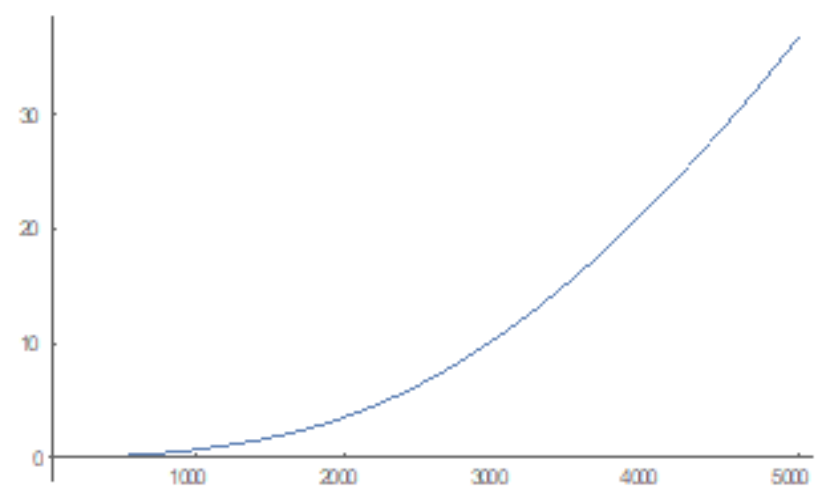

Figure 1: Dependence of Spectral Density on the Ku parameter. (See text for Definition of Ku.) 


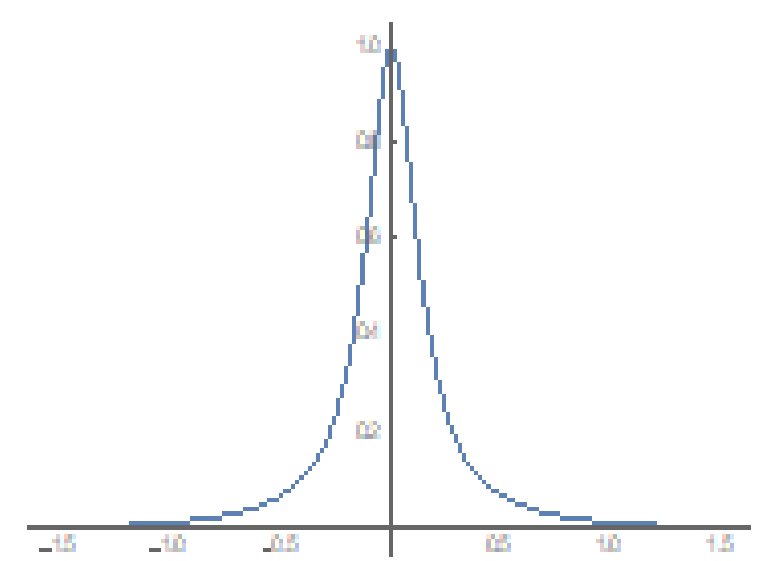

Figure 2: Plot of Probability Density Function Theta

\section{References Références Referencias}

1. O. H. J. Grohn, M. I. Kettuen, H. I. Makela, M. Penttonen, A. Pitkanen, J.A. Lukkarinen, R.A. Kauppinen, Early detection of irreversible cerebral ischemia in the rat using dispersion of the magnetic resonance imaging relaxation time, T1rho, $\mathrm{J}$ of Cerebral Blood Flow 20 (2000) 1457-66.

2. J. M. Hakumaki, O. H. Grohn, K, Tyynela, P. Valonen, S. Yla-Herttuala, R.A. Kauppinen, Early gene therapy-induced apoptotic response in BT4C gliomas by magnetic resonance relaxation contrast $\mathrm{TI}$ in the rotating frame, Cancer Gene Ther 9(4) (2002) 338-45.

3. M. I. Kettunen, A. M. J. Narvainen, P. K. Valonen, S. Yla-Herttua1a, R. A. Kauppinen, O. H. Grohn, Low spin-lock field $\mathrm{TI}$ relaxation in the rotating frame as a sensitive MR imaging marker for gene therapy treatment response in rat glioma, Radiology 243(3) (2007) 796-803.

4. E. Sitnitsky, Exactly solvable master equation for rotational reorientations of molecules, Recent Res. Devel. Chem. Physics, 7, (2013) 1-21

5. A. Abragam, Principles of Nuclear Magnetism, Clarendon Press, Oxford, 1991.

6. J.Blicharski, Nuclear magnetic relaxation in the rotating frame, Acta Phys Poi A 41 (1972).

7. T. Liimatainen, H. Hakkarainen, S. Mangia, J. M. Huttenun, C. Storino, D. Idiyatullin., D. Sorce, M. Garwood, S. Michaeli, MRI contrasts in high rank rotating frames, Magnetic Res. Medicine, Society of Magnetic Resonance in Medicine 73 (2015) 254-62

8. D. J. Sorce, S. Mangia, T. Liimatainen, M. Garwood, S. Michaeli, Exchange-induced relaxation in the presence of a fictitious field, Journal of magnetic resonance 245 (2014) 12-.[11]T.

9. Liimatainen, M. J. Nissi, M. T. Nieminen, S, Michaeli, M. Garwood, H. Grohn, Relaxation Along Fictitious Field (RAFF) Contrast in Bovine Articular Cartilage.
10. Proceedings 18th Scientific Meeting and Exhibition of the International Society for Magnetic Resonance in Medicine (ISMRM), Stockholm, Sweden I - 7 May 2010 (2010).

11. W. T. Coffey, Y. P. Kalmykov, the Langevin Equation, World Scientific New Jersey, 2017.

12. A. C. Redfield, Adv. Magn. Reson. 10) (1965).

13. C. Slichter, Principles of magnetic resonance, Berlin, Heidelberg, New York Springer-Verlag; 1996.

14. M. Goldman, Formal theory of spin-lattice relaxation, J. Mag. Res. 149 (2001) 160-87.

15. R. Y. Dong, Relaxation and dynamics of molecules in the liquid crystalline phases, Prog. NMR Spec. 41 (2002) 115-151.

16. A. E. Sitnitsky, Anomalous diffusion coefficient in disordered media from NMR Relaxation, arXiv: 0709.0341v2 [cond-mat.soft] 13 Nov 2008. 